\title{
The first water fountain in a planetary nebula
}

Olga Suárez ${ }^{1}$, José Francisco Gómez ${ }^{2}$, Philippe Bendjoya ${ }^{1}$, Luis. F. Miranda ${ }^{3,4}$, Martín. A. Guerrero ${ }^{2}$, Gerardo Ramos-Larios ${ }^{5}$, J. Ricardo Rizzo ${ }^{6}$, and Lucero Uscanga ${ }^{2,6,7}$

${ }^{1}$ Lab. Lagrange, UMR7293, UNSA, CNRS, Observatoire de la Côte d'Azur, F-06300 Nice, France

${ }^{2}$ Instituto de Astrofísica de Andalucía (IAA-CSIC), Glorieta de la Astronomía, s/n, E-18008 Granada, Spain

${ }^{3}$ Departamento de Física Aplicada, Facultade de Ciencias, Campus Lagos-Marcosende s/n, Universidade de Vigo, E-36310 Vigo, Spain (present address)

${ }^{4}$ Consejo Superior de Investigaciones Científicas (CSIC), c/ Serrano, 117, E-28006 Madrid, Spain

${ }^{5}$ Instituto de Astronomía y Meteorología, Av. Vallarta No. 2602, Col. Arcos Vallarta, 44130 Guadalajara, Jalisco, Mexico

${ }^{6}$ Centro de Astrobiología (INTA-CSIC), Ctra. M-108, km. 4, E-28850 Torrejón de Ardoz, Spain ${ }^{7}$ Observatorio Astronómico Nacional (IGN), E-28014 Madrid, Spain

\begin{abstract}
Water fountains are evolved stars showing water masers with velocity spanning more than $\sim 100 \mathrm{~km} / \mathrm{s}$. They usually appear at the end of the Asymptotic Giant Branch (AGB) phase or at the beginning of the post-AGB phase, and their masers trace the first manifestation of axisymmetric collimated mass-loss. For the first time, masers with water fountain characteristics have been detected towards a PN (IRAS 15103-5754), which might require a revision of the current theories about jet formation and survival times. IRAS 15103-5754 was observed using the ATCA interferometer at $22 \mathrm{GHz}$ (both continuum and water maser). The main results of these observations are summarized here. The evolutionary classification of this object is also discussed.
\end{abstract}

Keywords. masers, stars: AGB and post-AGB, stars: evolution, ISM: planetary nebulae: individual (IRAS 15103-5754)

\section{Introduction}

Water fountains are evolved stars showing water masers with high velocity components $\left(\geqslant 100 \mathrm{~km} \mathrm{~s}^{-1}\right)$. The name "water fountain" was first used by Likkel (1989) to refer to IRAS 16342-3814. In the last 10 years, the number of known water fountains has increased to more than 10 (see the review by J.F. Desmurs in these proceedings). The importance of these objects lies on the jets that are traced by their water maser emission. These jets could be the first manifestation of axisymmetric, collimated emission in evolved stars.

According to Sahai \& Trauger (1998), the shapping of bipolar/multipolar planetary nebulae $(\mathrm{PNe})$ is due to the presence of jets produced during the post-Asymptotic Giant Branch (post-AGB) phase. The mechanism that drives these jets is still unknown. Water fountains are probably the best objects to study the onset of these jets, since their dynamical ages are shorter than $100 \mathrm{yr}$ (see, for example Imai et al. 2002, 2007).

All the water fountains discovered up to now have been found in either post-AGB or late AGB stars. The mechanism driving the jet is believed to act during the post-AGB phase (Imai et al. 2007) and, water masers are expected to survive up to a maximum of 
$\simeq 100 \mathrm{yr}$ after the end of the AGB mass-loss (Gómez et al. 1990; Lewis et al. 1990). Therefore, if this scenario is correct, the presence of water fountain characteristics in a $\mathrm{PN}$ is extremely unlikely.

During a single-dish survey for water masers in evolved stars (Suárez et al. 2009), we detected water masers with high velocity components $\left(\sim 80 \mathrm{~km} \mathrm{~s}^{-1}\right)$ towards a PN candidate: IRAS 15103-5754 (I15103, hereafter). The velocity spread of the water maser components makes I15013 the first PN - water fountain candidate.

\section{Observations and results}

To confirm the physical association between water maser emission and I15103 and to confirm the PN nature of this source, we simultaneously observed water maser emission and radio continuum (both at $\simeq 22 \mathrm{GHz}$ ), using the Australia Telescope Compact Array on August 2011. We self-calibrated the maser emission, and then applied the same phase and amplitude correction to the continuum. Such procedure allowed us to obtain a very high relative positional accuracy between maser and continuum emission, with uncertainties $<50$ mas. We found the maser and radio continuum emission to be spatially coincident, thus confirming their association.

The water maser spectrum and the spatial distribution of the different components are shown in Fig. 1. The phase center of these observations is R.A.(J2000) $=15^{h} 14^{m} 18.4^{s}$, $\operatorname{Dec}(\mathrm{J} 2000)=-58^{\circ} 05^{\prime} 21.0^{\prime \prime}$. By "maser components" we refer to individual intensity peaks in the spectrum. The positional information has been obtained by fitting elliptical gaussians to the maser components, only in the channels in which a spectral peak is present. The total velocity span of the maser emission is $\simeq 80 \mathrm{~km} \mathrm{~s}^{-1}$, from $V_{\mathrm{LSR}} \simeq-70$ to $+10 \mathrm{~km} \mathrm{~s}^{-1}$.

There has been an important increase in the maximum flux density of the water maser components with respect to the spectrum obtained in 2007 with the Parkes telescope (Suárez et al. 2007). In 2007, the maximum flux density was observed at $V_{\mathrm{LSR}} \sim-37 \mathrm{~km} \mathrm{~s}^{-1}$ and it reached $\sim 60 \mathrm{Jy}$. In 2011 the maximum flux density was also observed at $V_{\mathrm{LSR}} \sim-37 \mathrm{~km} \mathrm{~s}^{-1}$ but reaching $\sim 1700 \mathrm{Jy}$. Thus, this source displays the most variable water maser emission among all known water fountains. However, we note that the global velocity span of the maser components has not changed significantly between 2007 and 2011 .

\section{Discussion}

\subsection{The nature of the source}

This source was classified as a PN mainly based on the presence of radio continuum emission (van de Steene \& Pottasch 1993). In principle, a young stellar object (YSO) could show both radio continuum and water maser emission, but there are reasons to rule out this interpretation for I15103:

- High density tracers detected toward this source are weak $\left(T_{a}<0.2 \mathrm{~K}\right)$, as seen in the CS and $\mathrm{NH}_{3}$ spectra from the RMS (Urquhart et al. 2008) and HOPS (Walsh et al. 2011) surveys, respectively. Strong emission of these tracers are normally associated with active YSOs (Anglada et al. 1989, 1996)

- Moreover, molecular lines detected towards I15103 are narrow $\left(\simeq 0.6 \mathrm{~km} \mathrm{~s}^{-1}\right.$ for CS, Urquhart et al. 2008), which is not consistent with arising from the environment around a YSO, specially if the object is so active that it pumps water maser emission. This would induce significant heating and turbulence in the gas, thus widening the molecular 
lines $\left(\Delta V \geqslant 2 \mathrm{~km} \mathrm{~s}^{-1}\right.$, Anglada et al. 1996). Therefore, it seems that the CS and $\mathrm{NH}_{3}$ emission traces dense molecular gas that is not associated with this source.

- If the source were a YSO out of the parental cloud, it would be detected at optical wavelengths, but this is not the case.

Assuming that I15103 is an evolved object, the reasons that support its classification as a PN are:

- Presence of radio continuum emission, which indicates ionisation.

- IR images show a bipolar morphology, consistent with that observed in developed PN (Lagadec et al. 2011; Ramos-Larios et al. 2012)

- The detection of significant [NeII] emission at $12.8 \mu \mathrm{m}$ (M.Blanco, private communication), typically associated to photoionisation. This implies that I15103 has reached the temperature necessary to become a PN.

\subsection{The morphology of the source}

The water maser components in Fig. 1 show a different spatial distribution from that typically found in the rest of water fountains, where they follow a bipolar pattern. In this case, there seems to be a trend, with more blueshifted water maser components towards the NW, while the more redshifted ones are located to the SE. However, it is difficult to define a clear bipolar structure in the water maser distribution, and its preferential orientation.

The mid-IR images of this PN obtained by Lagadec et al. (2011) with VISIR at the VLT show a bipolar structure oriented NE - SW, in nearly the same direction as the nearIR images shown in Ramos-Larios et al. (2012), taken with the NTT telescope at La Silla
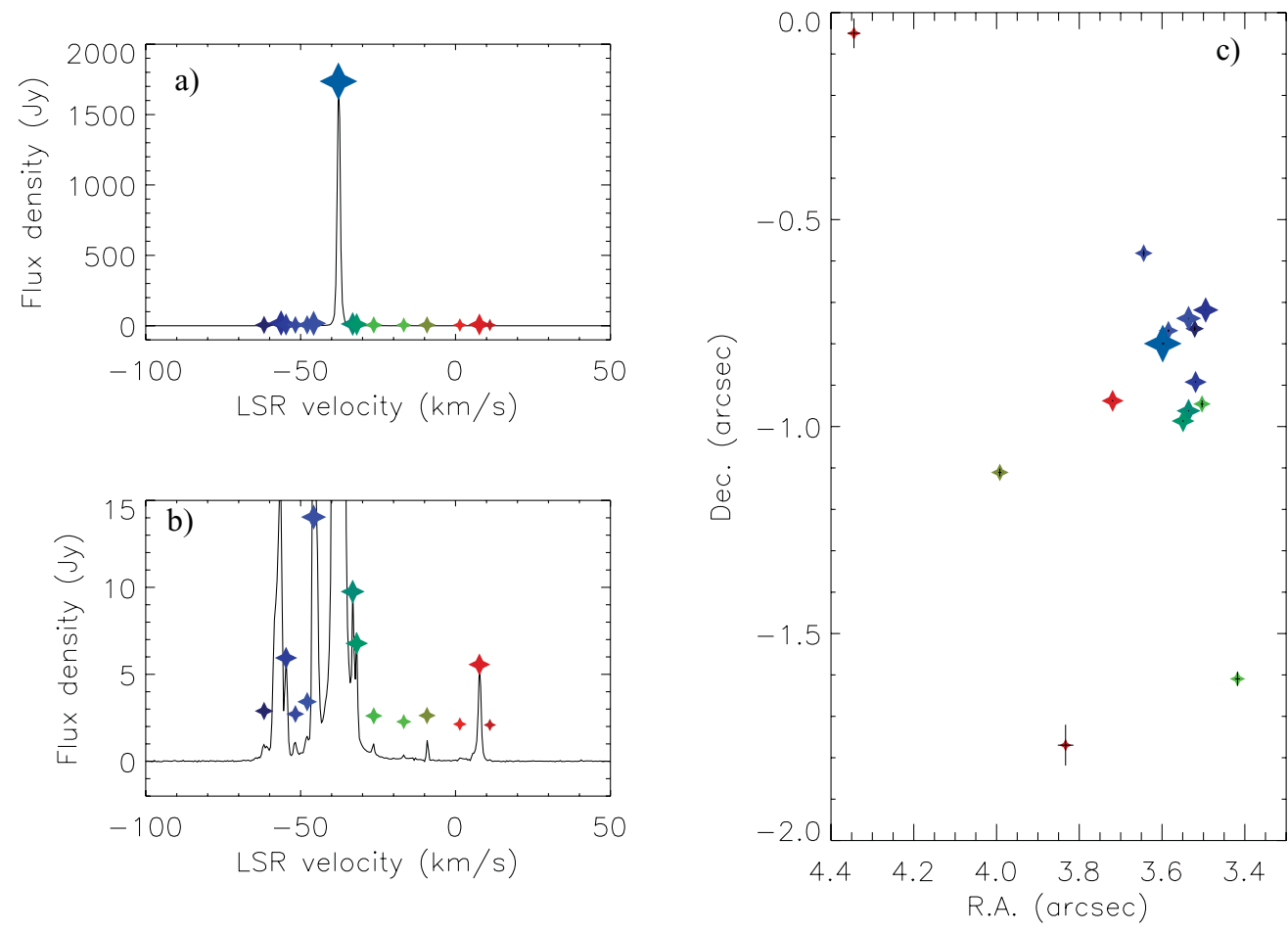

Figure 1. Spectrum (a, b) and spatial distribution (c) of the water maser components towards I15103. A zoom of the spectrum is shown in b) to display the weaker components. Symbols are proportional to the logarithm of the flux density of the individual maser components 


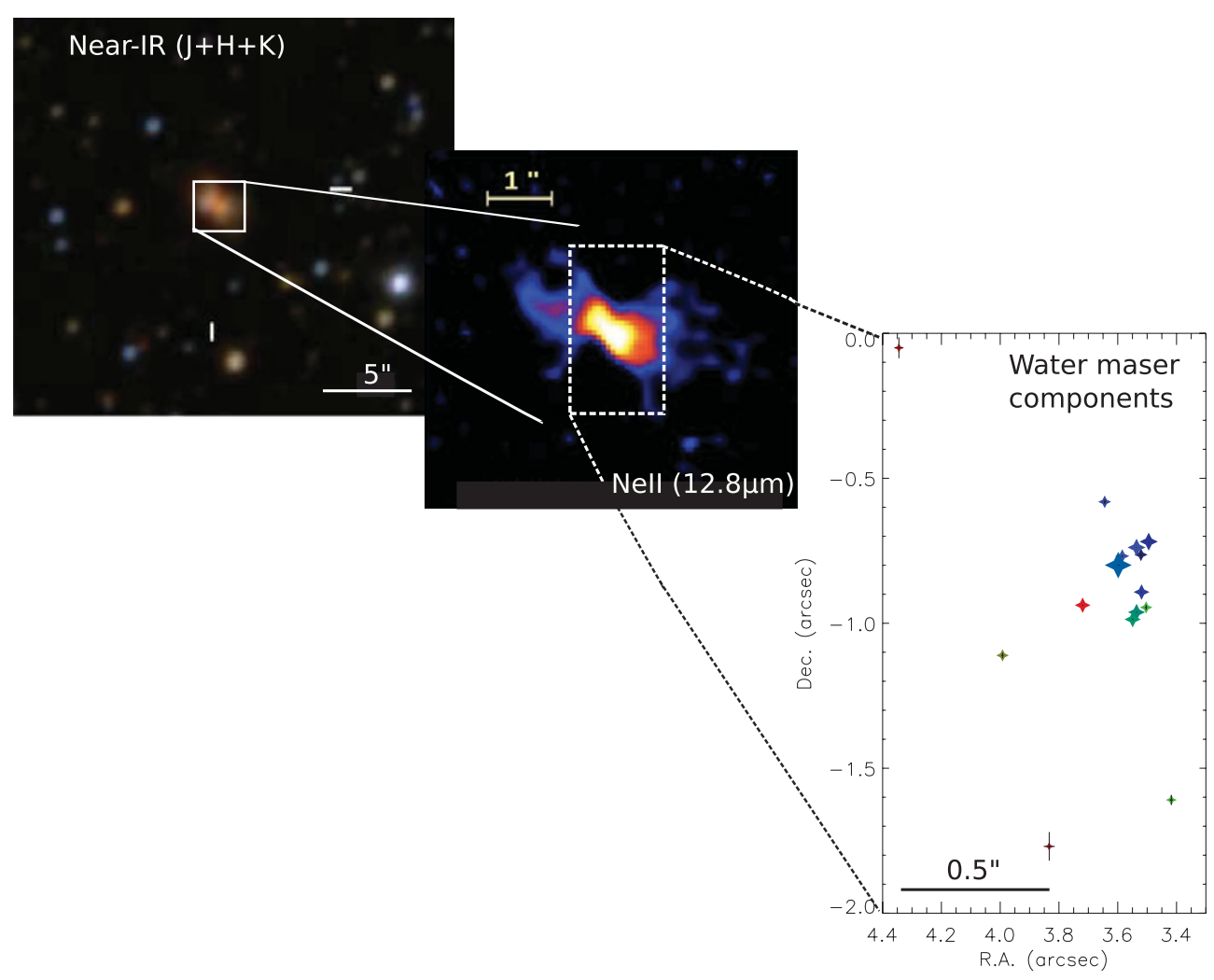

Figure 2. Left: near-IR image from Ramos-Larios et al. (2012); center: mid-IR image from Lagadec et al. (2011); right: water maser distribution

(Chile). These images are shown in Fig. 2 in comparison with the spatial distribution of the masers. The maser emission does not seem to follow the orientation of the IR images. In order to understand the real orientation of the maser emission with respect to the structure of the nebula, the information about the proper motion of the masers would be helpful.

\subsection{The evolutionary scenario for water fountain-PNe}

The discovery of the first PN with water fountain characteristics leads us to revise the scenario for the evolution of evolved sources harbouring water masers. This source is the fourth PN found showing water maser emission, but the other three: K3-35 (Miranda et al. 2001), IRAS 17347-3139 (de Gregorio-Monsalvo et al. 2004) and IRAS 18061-2505 (Gómez et al. 2008), do not show high velocity maser components.

The presence of water maser jets (which typically show very short dynamical ages) in an already bipolar-shaped PN supports the idea that jets in water fountains could be a ballistic and repetitive phenomenon. Episodic jets, ejected from the central star, would be traced by the water masers. These jets could be first launched at the end of the AGB or the beginning of the post-AGB stages but, as we are witnessing, they could, in the most extreme cases, be also launched, or at least maintained, when the object has already become a PN. Moreover, the outflows traced by water masers in IRAS 15103 are completely misaligned with respect to the bipolar axis of symmetry, what could indicate a different outflow episode at a different orientation. 


\section{Conclusion}

We have shown the water maser distribution of the first known PN with water fountain characteristics. The existence of such a source supports the idea that the jets of water fountains are ejected as successive episodes.

\section{References}

Anglada, G., Estalella, R., Pastor, J., Rodriguez, L. F., \& Haschick, A. D. 1996, ApJ, 463, 205 Anglada, G., Rodriguez, L. F., Torrelles, J. M., et al. 1989, ApJ, 341, 208

de Gregorio-Monsalvo, I., Gómez, Y., Anglada, G., et al. 2004, ApJ, 601, 921

Gómez, J. F., Suárez, O., Gómez, Y., et al. 2008, AJ, 135, 2074

Gómez, Y., Moran, J. M., \& Rodríguez, L. F. 1990, Rev. Mexicana AyA, 20, 55

Imai, H., Obara, K., Diamond, P. J., Omodaka, T., \& Sasao, T. 2002, Nature, 417, 829

Imai, H., Sahai, R., \& Morris, M. 2007, ApJ, 669, 424

Lagadec, E., Verhoelst, T., Mekarnia, D., et al. 2011, $A \mathscr{E} A$ in press

Lewis, B. M., Eder, J., \& Terzian, Y. 1990, ApJ, 362, 634

Likkel, L. 1989, ApJ, 344, 350

Miranda, L. F., Gómez, Y., Anglada, G., \& Torrelles, J. M. 2001, Nature, 414, 284

Ramos-Larios, G., Guerrero, M., Suárez, O., Miranda, L. F., \& Gómez, J. F. 2012, Submitted to $A \mathscr{E} A$

Sahai, R. \& Trauger, J. T. 1998, AJ, 116, 1357

Suárez, O., Gómez, J. F., Miranda, L. F., et al. 2009, A\& $A$ 505, 217

Suárez, O., Gómez, J. F., \& Morata, O. 2007, A\& A, 467, 1085

Urquhart, J. S., Hoare, M. G., Lumsden, S. L., Oudmaijer, R. D., \& Moore, T. J. T. 2008, in Astronomical Society of the Pacific Conference Series, Vol. 387. Ed. H. Beuther, H. Linz, \& T. Henning, 381

van de Steene, G. C. M. \& Pottasch, S. R. 1993, A\&\&A, 274, 895

Walsh, A. J., Breen, S. L., Britton, T., et al. 2011, MNRAS, 416, 1764 